\title{
Thermodynamics of Hydrogen and Hydrogen-Helium Plasmas: Path Integral Monte Carlo Calculations and Chemical Picture
}

\author{
V. S. Filinov ${ }^{* 1}$, P. R. Levashov ${ }^{1}$, M. Bonitz ${ }^{2}$, and V. E. Fortov ${ }^{1}$ \\ ${ }^{1}$ Institute for High Energy Density, Russian Academy of Sciences, Izhorskaya 13/19, Moscow 125412, Russia \\ ${ }^{2}$ Christian-Albrechts-Universität zu Kiel, Institut für Theoretische Physik und Astrophysik, Leibnizstr. 15, \\ 24098 Kiel, Germany
}

Received 3 February 2005, accepted 20 March 2005

Published online 13 June 2005

Key words Path integral Monte Carlo, Chemical picture, Hydrogen-helium plasma, Plasma phase transition. PACS 52.25.Kn, 52.65.Pp

\begin{abstract}
In this paper we study thermodynamic properties of hydrogen and hydrogen-helium mixtures with the help of the direct path integral Monte Carlo simulations. The results are compared with available theoretical and experimental methods based, in particular, on chemical picture. We investigate the effects of temperature ionization in low-density hydrogen plasma. We also present a number of calculated isotherms for hydrogenhelium mixture with the mass concentration of helium $Y=0.234$ in the range from $10^{4} \mathrm{~K}$ to $2 \cdot 10^{5} \mathrm{~K}$. In the density region where a sharp conductivity rise have been observed experimentally the simulations give indications for one or two plasma phase transitions, in accordance with earlier theoretical predictions.
\end{abstract}

(C) 2005 WILEY-VCH Verlag GmbH \& Co. KGaA, Weinheim

\section{Introduction}

Many astrophysical problems require the knowledge of thermodynamic properties of hydrogen and helium [1-5]. To understand different effects in stellar structure and evolution one should provide accurate modelling of the underlying physics including equation of state (EOS) effects. In normal stars where plasma is fully-ionized and almost ideal the construction of EOS doesn't reveal particular difficulties. However the investigation of the giant planets Jupiter and Saturn, and to a lesser extent brown dwarfs demands thermodynamic information for hydrogen and helium in the approximate range of temperatures $10^{3} \mathrm{~K}<T<10^{5} \mathrm{~K}$ and mass densities $0.01<$ $\rho<100 \mathrm{~g} / \mathrm{cm}^{3}$. In this region the complexity of an EOS calculation increases considerably when nonideal effects are compounded with chemical reactions associated with partial pressure dissociation and ionization equilibria [6-10]. Moreover, in this region the the so-called plasma phase transition (PPT) has been predicted $[9,10]$. Significant efforts have been made in the last decades to understand the behaviour of dense fully-ionized and partially-ionized hydrogen and helium (see, for example, [8] and references therein). In these works mostly the chemical picture is applied for the calculation of thermodynamic properties. The chemical picture assumes that bound configurations, such as atoms and molecules, retain a definite identity and interact through pair potentials; in other words, this model is valid only at weak interparticle interactions. However at densities corresponding to pressure ionization the electrons in bound configurations become delocalized and bound species lose their definiteness [11]. Therefore there is a great interest in direct first-principle numerical simulations of strongly coupled degenerate systems which avoid such approximations.

In this work we use the direct path integral Monte Carlo (DPIMC) method to calculate the thermodynamic properties of hydrogen and hydrogen - helium mixtures. This method is well established theoretically and allows the treatment of quantum and exchange effects without any preliminary physical approximations. Using the results of our simulation we compare them with the model based on the chemical picture [1,2]. We also analyze the problem of plasma phase transition in dense hydrogen - helium mixtures and discuss several theoretical and experimental predictions of this phenomenon.

\footnotetext{
* Corresponding author: e-mail: filinov@ok.ru, Phone: +07 09593107 19, Fax: +07 0954857990
} 


\section{Direct path integral Monte Carlo}

Path integral Monte Carlo [12,14] is based upon Feynman's formulation of quantum-statistical mechanics using path integrals [15]. For hydrogen plasma one can find exhaustive description of the DPIMC elsewhere [13,14]. In this work we restrict ourselves by the brief explanation of ideas underlying the DPIMC for hydrogen-helium mixtures.

We consider the temperature range from $10^{4} \mathrm{~K}$ to $2 \cdot 10^{5} \mathrm{~K}$ and electron particle densities from $10^{20}$ to $3 \cdot 10^{24} \mathrm{~cm}^{-3}$. Under such conditions electrons are degenerate while protons and $\alpha$-particles can be treated as classical particles because of their relatively large masses. Thus for the case of electro-neutral hydrogen-helium plasma with volume $V$ the partition function $Z$ is given by

$$
Z\left(N_{e}, N_{p}, N_{\alpha}, V, \beta\right)=\frac{1}{N_{e} ! N_{p} ! N_{\alpha} !} \sum_{\sigma} \int_{V} d q_{p} d q_{\alpha} d r \rho\left(q_{p}, q_{\alpha}, r, \sigma ; \beta\right)
$$

Here $N_{e}, N_{p}$, and $N_{\alpha}$ are the numbers of electrons, protons, and $\alpha$-particles, correspondingly, $\beta=1 / k_{B} T, T$ is the temperature, $q_{p} \equiv\left\{\mathbf{q}_{p 1}, \mathbf{q}_{p 2}, \cdots, \mathbf{q}_{p N_{p}}\right\}, q_{\alpha} \equiv\left\{\mathbf{q}_{\alpha 1}, \mathbf{q}_{\alpha 2}, \cdots, \mathbf{q}_{\alpha N_{\alpha}}\right\}$ are the coordinates of protons and $\alpha$-particles, respectively, $r \equiv\left\{\mathbf{r}_{1}, \mathbf{r}_{2}, \cdots, \mathbf{r}_{N_{e}}\right\}$ are the coordinates of electrons, and $\sigma \equiv\left\{\sigma_{1}, \sigma_{2}, \cdots, \sigma_{N_{e}}\right\}$ are the spin variables of the electrons. The density matrix in (1) is expressed via a path integral:

$$
\begin{gathered}
\int_{V} d R^{(0)} \sum_{\sigma} \rho\left(R^{(0)}, \sigma ; \beta\right)=\sum_{P} \sum_{\sigma}(-1)^{\kappa_{P}} \int_{V} d R^{(0)} \cdots d R^{(n)} \times \\
\left.\rho^{(1)} \rho^{(2)} \cdots \rho^{(n)} S\left(\sigma, \hat{P} \sigma^{\prime}\right) \hat{P}\right) \rho^{(n+1)}
\end{gathered}
$$

where $\rho^{(i)}=\rho\left(R^{(i-1)}, R^{(i)} ; \Delta \beta\right) \equiv\left\langle R^{(i-1)}\left|e^{-\Delta \beta \hat{H}}\right| R^{(i)}\right\rangle, \Delta \beta \equiv \beta /(n+1), \hat{P}$ is the permutation operator, $\kappa_{P}$ is the parity of permutation, $S$ is the spin matrix, $\hat{H}$ is Hamiltonian of the system, $\hat{H}=\hat{K}+\hat{U}_{C}, \hat{K}$ is the kinetic energy, $\hat{U}_{C}$ is the potential energy, consisting of Coulomb interaction of electrons $(e)$, protons $(p)$, and $\alpha$-particles $(\alpha): \hat{U}_{C}=\hat{U}_{C}^{p}+\hat{U}_{C}^{e}+\hat{U}_{C}^{\alpha}+\hat{U}_{C}^{e p}+\hat{U}_{C}^{e \alpha}+\hat{U}_{C}^{p \alpha}$. We denote particle coordinates as follows: $R^{(i)}=\left(q_{p}, q_{\alpha}, r_{i}\right), i=1, \cdots, n+1, R^{(0)} \equiv\left(q_{p}, q_{\alpha}, r\right), R^{(n+1)} \equiv R^{(0)}, \sigma^{\prime}=\sigma$. Thus electrons participating in the simulation are represented as fermionic loops with $n$ vertexes: $[R] \equiv\left[R^{(0)} ; R^{(1)} ; \cdots ; R^{(n)} ; R^{(n+1)}\right]$. Exchange effects for Fermi statistics are taken into account by the permutation operator $\hat{P}$ and the sum over the permutations with parity $\kappa_{P}$. It is possible to reduce the expression (2) to a form in which the sum over all permutations is replaced by the determinant of the exchange matrix $\psi_{a b}^{n, 1}$. This technique allows us to improve the accuracy of simulation for strongly degenerate plasma:

$$
\begin{aligned}
& \sum_{\sigma} \rho\left(q_{p}, q_{\alpha}, r, \sigma ; \beta\right)=\frac{1}{\lambda_{p}^{3 N_{p}} \lambda_{\alpha}^{3 N_{\alpha}} \lambda_{\Delta}^{3 N_{e}}} \sum_{s=0}^{N_{e}} \rho_{s}([R], \beta), \\
& \rho_{s}([R], \beta)=\frac{C_{N_{e}}^{s}}{2^{N_{e}}} \exp \{-\beta U([R], \beta)\} \prod_{l=1}^{n} \prod_{m=1}^{N_{e}} \phi_{m m}^{l} \operatorname{det}\left|\psi_{a b}^{n, 1}\right|_{s} .
\end{aligned}
$$

Here $\lambda_{p}^{2}=2 \pi \hbar^{2} \beta / m_{p}, \lambda_{\alpha}^{2}=2 \pi \hbar^{2} \beta / m_{\alpha}, \lambda_{\Delta}^{2}=2 \pi \hbar^{2} \Delta \beta / m_{e}, m_{p}, m_{\alpha}, m_{e}$ are the masses of proton, $\alpha$ particle and electron, respectively. In equation (3) $U=U^{p p}+U^{\alpha \alpha}+U^{p \alpha}+\sum_{l=1}^{n}\left\{U_{l}^{e e}+U_{l}^{e p}+U_{l}^{e \alpha}\right\} /(n+1)$ and $\phi_{m m}^{l} \equiv \exp \left[-\pi\left|\xi_{m}^{(l)}\right|^{2}\right]$ are the functions generated from the kinetic energy density matrix, $\xi^{(1)}, \cdots, \xi^{(n)}$ are the dimensionless distances between neighbor vertexes of fermionic loops which represent electrons $[R] \equiv$ $\left[R^{(0)} ; R^{(0)}+\lambda_{\Delta} \xi^{(1)} ; R^{(0)}+\lambda_{\Delta}\left(\xi^{(1)}+\xi^{(2)}\right) ; \cdots\right]$. Elements of the exchange matrix $\psi_{a b}^{n, 1}$ are defined by the expression:

$$
\left\|\psi_{a b}^{n, 1}\right\|_{s} \equiv\left\|\exp \left\{-\frac{\pi}{\lambda_{\Delta}^{2}}\left|\left(r_{a}-r_{b}\right)+y_{a}^{n}\right|^{2}\right\}\right\|_{s}, y_{a}^{n}=\lambda_{\Delta} \sum_{k=1}^{n} \xi_{n}^{(k)}
$$


The index $s$ stands for the number of electrons with same spin projection.

As a high-temperature density matrix one can use its asymptote in the limit $T \rightarrow \infty$. Every $N$-particle hightemperature density matrix is represented as a product of two-particle density matrices. For the two-particle density matrix there is an analytical solution of the Bloch equation by first-order perturbation theory [17]:

$$
\begin{aligned}
\rho\left(\mathbf{r}_{a}, \mathbf{r}_{a}^{\prime}, \mathbf{r}_{b}, \mathbf{r}_{b}^{\prime}, \beta\right)= & \\
& \frac{m_{a} m_{b}}{\left(2 \pi \hbar^{2} \beta\right)^{3}} \exp \left[-\frac{m_{a}}{2 \hbar^{2} \beta}\left(\mathbf{r}_{a}-\mathbf{r}_{a}^{\prime}\right)^{2}\right] \exp \left[-\frac{m_{b}}{2 \hbar^{2} \beta}\left(\mathbf{r}_{b}-\mathbf{r}_{b}^{\prime}\right)^{2}\right] \exp \left[-\beta \Phi^{a b}\right],
\end{aligned}
$$

where $\Phi^{a b}\left(\mathbf{r}_{a}, \mathbf{r}_{a}^{\prime}, \mathbf{r}_{b}, \mathbf{r}_{b}^{\prime}, \beta\right)$ - nondiagonal effective two-particle pseudopotential:

$$
\Phi^{a b}\left(\mathbf{r}_{a b}, \mathbf{r}_{a b}^{\prime}, \beta\right)=e_{a} e_{b} \int_{0}^{1} \frac{d \alpha}{d_{a b}(\alpha)} \operatorname{erf}\left(\frac{d_{a b}(\alpha}{2 \lambda_{a b} \sqrt{\alpha(1-\alpha)}}\right) .
$$

Here $d_{a b}(\alpha)=\left|\alpha \mathbf{r}_{a b}+(1-\alpha) \mathbf{r}_{a b}^{\prime}\right|, 0 \leq \alpha \leq 1, \operatorname{erf}(x)=2 / \sqrt{\pi} \int_{0}^{x} \exp \left(-t^{2}\right) d t$ denotes the error function, $\lambda_{a b}^{2}=\hbar^{2} \beta / 2 \mu_{a b}, e_{a}, e_{b}$ are the charges of particles, $m_{a}, m_{b}$ are the masses of particles, $\mu_{a b}^{-1}=m_{a}^{-1}+m_{b}^{-1}$ is the reduced mass. In the high-temperature limit two-particle nondiagonal effective potential (4) can be approximated by a half-sum of diagonal pseudopotentials:

$$
\Phi^{a b}\left(\left|\mathbf{r}_{a b}\right|, \Delta \beta\right)=\frac{e_{a} e_{b}}{\lambda_{a b} x_{a b}}\left\{1-\exp \left(-x_{a b}^{2}\right)+\sqrt{\pi} x_{a b}\left[1-\operatorname{erf}\left(x_{a b}\right]\right\}\right.
$$

where $x_{a b}=\left|\mathbf{r}_{a b}\right| / \lambda_{a b}$. It is worth to underline that $\Phi^{a b}\left(\left|\mathbf{r}_{a b}\right|, \Delta \beta\right)$ tends to a finite value at $x_{a b} \rightarrow 0$ and to the Coulomb potential $e_{a} e_{b} / x_{a b}$ at $x_{a b} \rightarrow \infty$. It is proved that the pseudopotential (5) coincides with an exact quantum potential at temperatures $T>2 \cdot 10^{5} \mathrm{~K}$ [18]. Thus, $U^{a a}$ and $U_{l}^{a b}$ in Eq. (3) are sums of the effective quantum pair interactions described by the $\Phi^{a b}$ between two charged plasma particles $a$ and $b$.

All thermodynamic properties can be expressed through the partition function derivatives. For example, pressure and total energy are given by the formulas:

$$
\begin{aligned}
& E=-\beta \partial \ln Z / \partial \beta \\
& \beta P=\partial \ln Q / \partial V=[\eta / 3 V \partial \ln Q / \partial \eta]_{\eta=1} .
\end{aligned}
$$

Multiple integrals in (6) are calculated with the help of the standard Metropolis technique in a cubic cell with periodic boundary conditions [12]. The accuracy $\epsilon$ of calculations depends on the number of factors $n$ in the equation (2), temperature $T$ and electron degeneracy parameter $\chi=n_{e} \lambda_{e}^{3}$ and is given by the expression $\epsilon \sim$ $(\beta \mathrm{Ry})^{2} \chi /(n+1)$, where $n_{e}$ is the particle density of electrons, $\lambda_{e}^{2}=2 \pi \hbar^{2} \beta / m_{e}, \mathrm{Ry}=e^{2} / 2 a_{B} \approx 13.6 \mathrm{eV}$. According to this estimation to simulate a Coulomb system at temperature $10^{4} \mathrm{~K}$ it is sufficient to choose $n=20$. High temperature density matrix in Eq. (2) relates in this case to temperature higher than Ry.

\section{Simulation results}

We tested our computational scheme by many ways. First of all we calculated thermodynamic properties of ideal hydrogen plasma [20] and found very good agreement up to degeneracy parameter $\chi=10$. To extend the range of $\chi$ to higher values we improved the treatment of exchange effects. Unlike the previous version of the method [12] in this work we take into account exchange effects not only inside the main Monte Carlo cell but also in the neighboring periodic images. It is necessary to include such procedure into the algorithm if the electron thermal wavelength is comparable or larger than the size of the Monte Carlo cell. Thus the exchange interaction was calculated in the nearest $3^{3}, 5^{3}$ etc. Monte Carlo cells in accordance with the value of electron thermal 
wavelength. The accuracy of exchange effects treatment was controlled by comparing the results of calculations with analytical dependencies for pressure and energy of ideal degenerate plasma.

We also studied interacting hydrogen plasma in a wide range of temperatures and particle densities [21, 22]. The DPIMC method allowed us to investigate the effects of temperature and pressure dissociation and ionization $a b$ initio. From the analysis of pair distribution functions we observed the formation and break-up of molecules and atoms under different conditions. At very high density we also observed the effect of proton ordering indicating the formation of Coulomb crystal. We found rather good agreement with the calculations performed by other methods at small and medium densities. However at high values of plasma density in the region of pressure ionization no reliable analytical methods exist.

The simulation results for hydrogen plasma in the region of temperatures from $T=10^{4} \mathrm{~K}$ to $10^{6} \mathrm{~K}$ and electron particle densities from $n_{e}=10^{22} \mathrm{~cm}^{-3}$ to $10^{24} \mathrm{~cm}^{-3}$ allowed us to calculate the deuterium shock Hugoniot [23]. It is interesting to note that the resulting curve is located between the experimental data of Knudson et al. [24] and Collins et al. [25].

In this work we compare our simulation results with available data on hydrogen and hydrogen-helium mixture from Refs. [2,7]. This model is based on the chemical picture with classical statistics for molecules and ions and Fermi-Dirac statistics for the electrons. It takes into account a lot of physical effects including dissociation and ionization, interactions between charged particles and neutral atoms and molecules, neutral-neutral interactions, high-pressure screening effects, excited electronic states of molecules as well as a number of "second-order" phenomena. Owing to the complexity of the model [2,7] equations of state for hydrogen and helium are presented in tabular form [2]. So thermodynamic properties of pure hydrogen or helium can be calculated directly with the help of relatively simple interpolation code. The properties of hydrogen-helium mixtures can then be obtained by interpolation in composition between the two pure EOS. Using the so-called "linear mixing" technique it is possible to approximate density $\rho(P, T)$ of the hydrogen-helium mixture with the mass fraction of helium $Y$ at pressure $P$ and temperature $T$ :

$$
\frac{1}{\rho(P, T)}=\frac{1-Y}{\rho^{\mathrm{H}}(P, T)}+\frac{Y}{\rho^{\mathrm{He}}(P, T)} .
$$
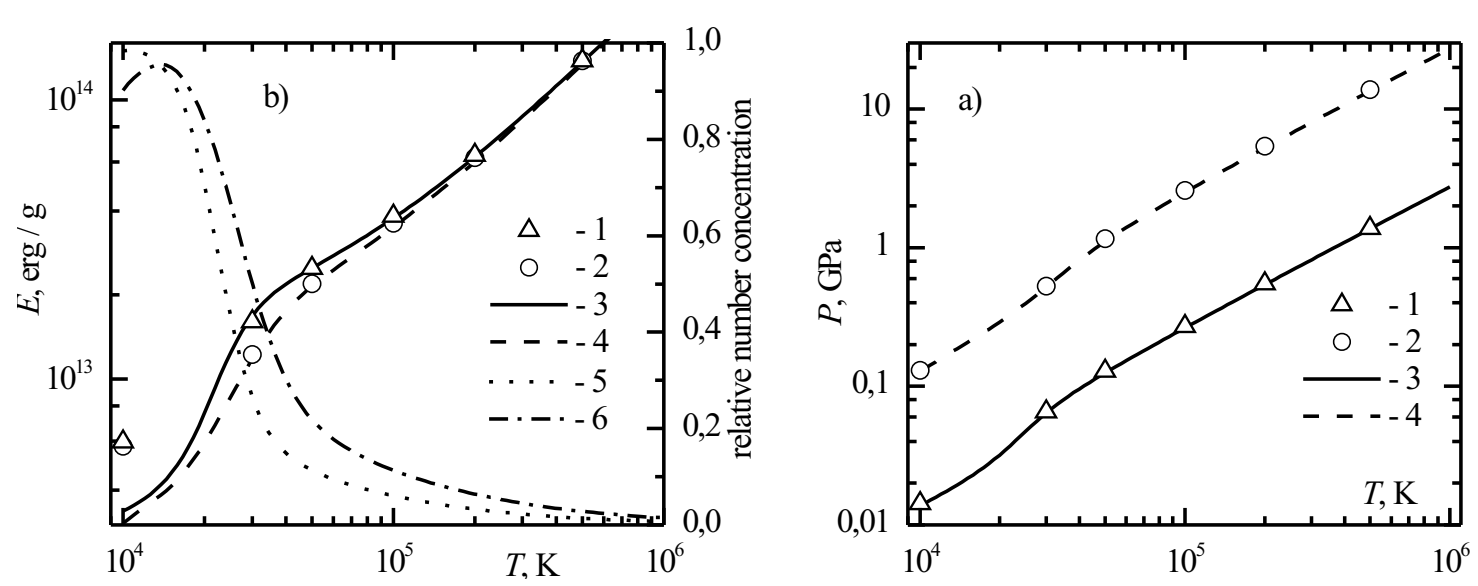

Fig. 1 The DPIMC (triangles and circles) and theoretical (solid and dashed lines) [2,7] isochors for pressure (a) and energy (b) in hydrogen plasma vs. temperature. Calculations: $1-$ isochor $n_{e}=10^{20} \mathrm{~cm}^{-3} ; 2-$ isochor $n_{e}=10^{21} \mathrm{~cm}^{-3}$. EOS calculations [2]: 3 - isochor $n_{e}=10^{20} \mathrm{~cm}^{-3} ; 4-$ isochor $n_{e}=10^{21} \mathrm{~cm}^{-3} ; 5,6$ - relative number concentration of hydrogen atoms (b, right axis) along the isochor $n_{e}=10^{20} \mathrm{~cm}^{-3}$ and $n_{e}=10^{21} \mathrm{~cm}^{-3}$, correspondingly

In Fig. 1 it is shown pressure and energy dependencies vs. temperature along two isochors $n_{e}=10^{20}$ and $10^{21} \mathrm{~cm}^{-3}$ of hydrogen plasma. The agreement with hydrogen EOS [2] is very good for pressure at all temperatures (Fig. 1a) and for energy at temperatures higher than $3 \cdot 10^{4} \mathrm{~K}$ (Fig. 1b). The relative number concentration of hydrogen atoms shown in Fig. $1 \mathrm{~b}$ by dash-dotted and dotted lines indicate that hydrogen at $T=10^{4} \mathrm{~K}$ and 
$n_{e}=10^{20} \div 10^{21} \mathrm{~cm}^{-3}$ consists mainly of atoms. The fact that at $10^{4} \mathrm{~K}$ in our calculations we have higher absolute energy values than in the theoretical model can be connected with the difficulties of correct interpretation of bound states both in the DPIMC and in chemical picture. Therefore this problem needs further investigation.
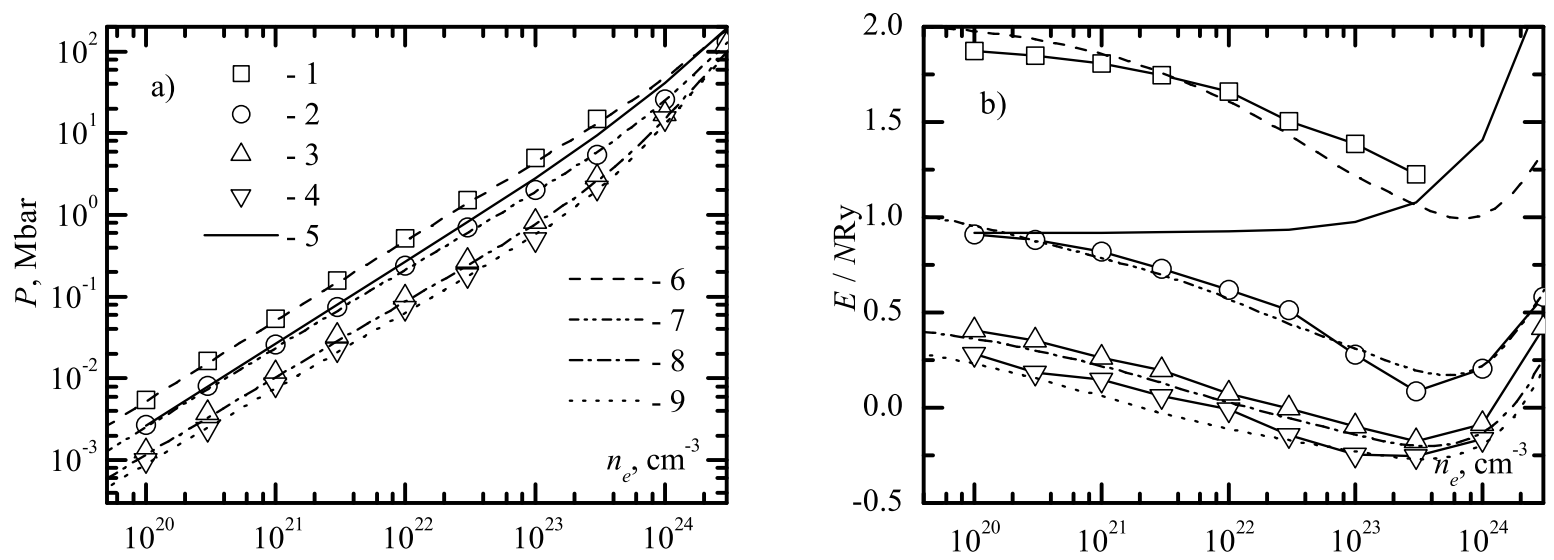

Fig. 2 Pressure (a) and energy (b) in a hydrogen-helium mixture with the mass concentration of helium $Y=0.234$. Shown are isotherms calculated with the help of DPIMC method (symbols and symbols with lines) and related isotherms computed with the help of EOS [2,7] (lines). DPIMC ( $[2,7])$ calculations: $1(6)-200 \mathrm{kK}, 2(7)-100 \mathrm{kK}, 3(8)-50 \mathrm{kK}, 4(9)-$ $40 \mathrm{kK} .5-100 \mathrm{kK}$ isotherm for ideal plasma.

Besides hydrogen plasma, we also carried out calculations of thermodynamic properties of hydrogen-helium mixture with a composition corresponding to that of the outer layers of the Jovian atmosphere. During the mission of the Galileo spacecraft the helium abundance in the atmosphere of Jupiter was determined as $Y=$ $m_{\mathrm{He}} /\left(m_{\mathrm{He}}+m_{\mathrm{H}}\right)=0.234$ and was close to the present-day protosolar value $Y=0.275$. As the model of the Jupiter is significantly determined by its composition and EOS, it was interesting to simulate the thermodynamic properties of the mixture with such composition in the region of pressure dissociation and ionization where traditional chemical models of plasma fail.
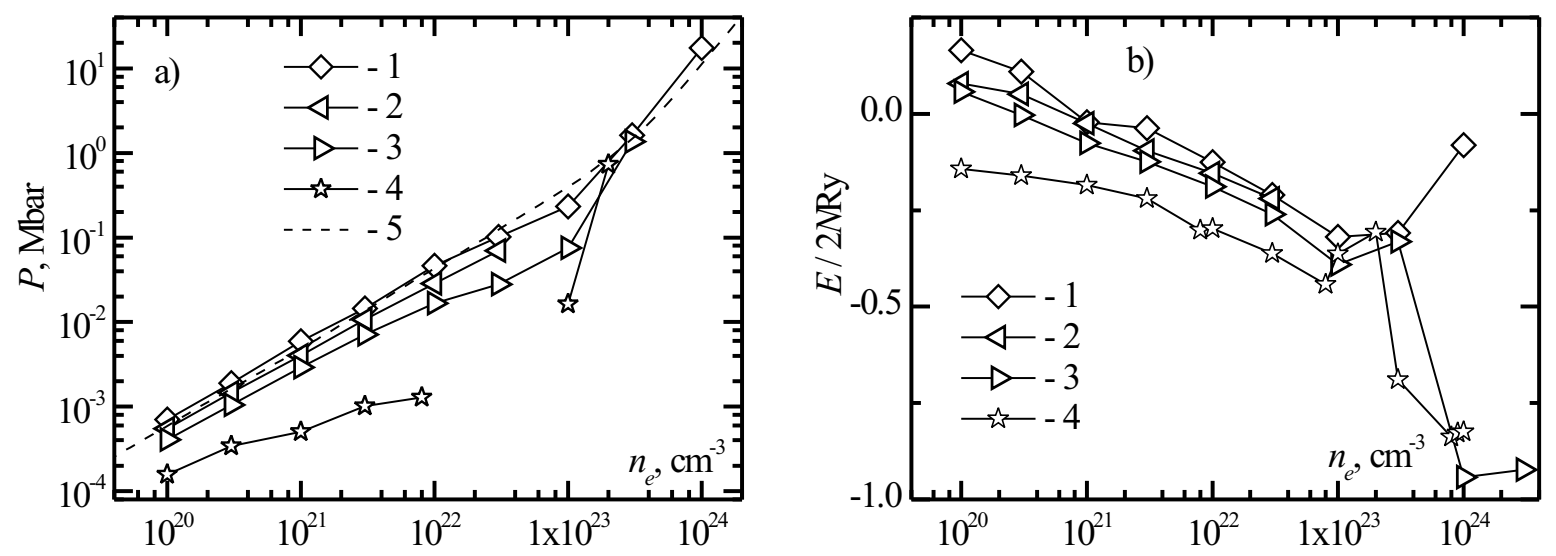

Fig. 3 Pressure (a) and energy (b) in a hydrogen-helium mixture with the mass concentration of helium $Y=0.234$. Shown are isotherms calculated with the help of DPIMC method (symbols with lines). DPIMC calculations: $1-30 \mathrm{kK}, 2-25 \mathrm{kK}$, $3-20 \mathrm{kK}, 4-10 \mathrm{kK}$. EOS calculations [2,7] (a): $5-30 \mathrm{kK}$

Calculations were fulfilled in the region of temperatures from $T=10^{4} \mathrm{~K}$ to $2 \cdot 10^{5} \mathrm{~K}$ and electron densities from $n_{e}=10^{20} \mathrm{~cm}^{-3}$ to $3 \cdot 10^{24} \mathrm{~cm}^{-3}$. The results of comparison are shown in Fig. 2 and 3. The agreement between our calculations and the model [2] along the isotherms $T=4 \cdot 10^{4}, 5 \cdot 10^{4}, 10^{5}$, and $2 \cdot 10^{5} \mathrm{~K}$ is quite good and becomes better with the increase of temperature. The smaller values of pressure on the DPIMC 
isotherm $3 \cdot 10^{4} \mathrm{~K}$ near the particle density value $10^{23} \mathrm{~cm}^{-3}$ can be explained by a strong influence of interaction and bound states in this region; these effects are taken into account only approximately in the model [2]. The formation of atoms and molecules is also the reason of the pressure and energy reduction along the $10^{5} \mathrm{~K}$ isotherm with respect to the isotherm of non-interacting hydrogen-helium mixture (see Fig. 2).

In Fig. 3 the dependence of pressure and energy vs. electron density along isotherms are shown. At temperatures 30 and $25 \mathrm{kK}$ the isotherms have no peculiarities. However along the isotherm $T=2 \cdot 10^{4} \mathrm{~K}$ there is a region for $n_{e}>3 \cdot 10^{23} \mathrm{~cm}^{-3}$, where the pressure strongly fluctuates and even becomes negative. Also along the isotherms $T=10^{4} \mathrm{~K}$ and $T=1.5 \cdot 10^{4} \mathrm{~K}$ there are two such regions $10^{22} \mathrm{~cm}^{-3}<n_{e}<10^{23} \mathrm{~cm}^{-3}$ and $n_{e}>3 \cdot 10^{23} \mathrm{~cm}^{-3}$. Earlier we found a similar effect for pure hydrogen at $T=10^{4} \mathrm{~K}$ in the region of pressure ionization and showed that in the transition region a number of large clusters (droplets) were formed [26, 27]. In this region of pressure ionization the PPT was predicted by many authors $[6,7,9-11,28-30]$ and moreover a sharp electrical conductivity rise was measured in [31]. The instabilities in our calculations indicate the existence of PPT in dense hydrogen. Later [14] we found the PPT and the formation of clusters in electron-hole plasma of germanium semiconductor at low temperature and found good agreement with experimental phase diagram [32]. The presence of clusters (droplets) in plasma decreases the total energy of the system as can be seen in Fig. 3. The region of existence of bound states is determined by deep minima on isotherms. The analysis of pair distribution functions reveals that the part of hydrogen molecules increases with the temperature drop. At low temperatures $T=10^{4}$ and $2 \cdot 10^{4} \mathrm{~K}$ we can observe the formation of large clusters of atoms and molecules.
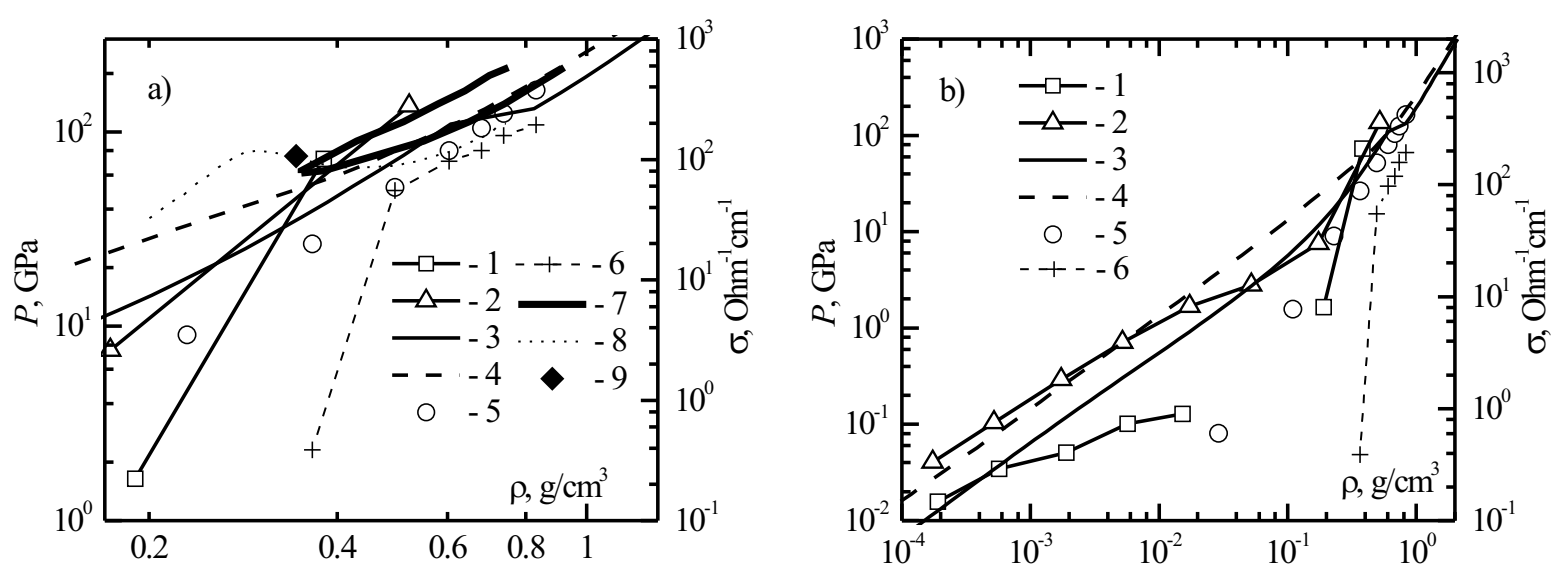

Fig. 4 The DPIMC (lines with squares and triangles) and theoretical $[2,7]$ isotherms for pressure in a hydrogen-helium mixture with the mass concentration of helium $Y=0.234 \mathrm{vs}$. density. DPIMC calculations: $1-10 \mathrm{kK}, 2-20 \mathrm{kK}$. EOS calculations: $3-10 \mathrm{kK}, 4-20 \mathrm{kK}$. Experiment [35]: 5 - quasi-isentrope of hydrogen-helium mixture, $T \sim 5000 \mathrm{~K}, 6-$ electrical conductivity of hydrogen-helium mixture along the quasi-isentrope (right axis). 7 - phase boundaries of PPT in hydrogen [2,7], 8 - undercritical metastable isotherm $T=1.2 \cdot 10^{4} \mathrm{~K}$ and $Y=0.308$ [30], 9 - critical point of PPT in hydrogen-helium mixture with $Y=0.308$ [30]

The problem of PPT in a hydrogen-helium mixture is significantly determined by the composition of the mixture $[2,7,30]$. From shock-wave experiments one can estimate the range of temperature and density where a sharp electrical conductivity rise takes place. In quasi-isentropic compression the transition from a low-conductivity state to a high-conductivity one for hydrogen occurs at $T \sim 3-15 \mathrm{kK}$ and $\rho \sim 0.4-0.7 \mathrm{~g} / \mathrm{cm}^{3}[31,33]$ whereas for helium at $T \sim 15-40 \mathrm{kK}$ and $\rho \sim 0.7-1.25 \mathrm{~g} / \mathrm{cm}^{3}$ [34]. However it is not enough to determine the region of existence of the PPT. According to theoretical equations of state the critical point of the PPT in pure hydrogen is $T_{c r_{\mathrm{H}}} \sim 12-19 \mathrm{kK}, P_{c r_{\mathrm{H}}} \sim 0.2-0.9$ Mbar [2, 7, 30,36]. In pure helium the critical point was found to be $T_{c r_{\mathrm{He}}} \sim 17 \mathrm{kK}$ [30] or $T_{c r_{\mathrm{He}}} \sim 35 \mathrm{kK}$ [36] and $P_{c r_{\mathrm{He}}} \sim 7$ Mbar [30,36]. For hydrogen-helium mixture with a mole fraction of hydrogen 0.93 the critical temperature was found to be $\sim 35 \mathrm{kK}$ [36], however the dependence of critical parameters on the plasma composition has not been investigated. In accordance with the quantum-statistical equation of state [30] at helium mass concentration $Y<0.93$ and temperature less than both critical temperatures the properties of hydrogen-helium mixture are determined mostly by hydrogen and only one PPT exists. At high values of $Y>0.93$ both the hydrogen and helium PPT can occur. In our DPIMC 
simulations we have observed one region of instability with negative pressure at $T=2 \cdot 10^{4} \mathrm{~K}$ and two regions of instability at $T=10^{4} \mathrm{~K}$ even at $Y=0.234$. The results of our simulation are shown in Fig. 4 together with experimental data and theoretical predictions.

Along the isotherm $T=2 \cdot 10^{4} \mathrm{~K}$ we found the region with bad convergence in the range of densities between 0.5 and $5 \mathrm{~g} / \mathrm{cm}^{3}$. Along the isotherms $T=1.5 \cdot 10^{4} \mathrm{~K}$ and $T=10^{4} \mathrm{~K}$ such region is even wider and begins from $0.38 \mathrm{~g} / \mathrm{cm}^{3}$. Surprisingly there was another region where pressure became negative: from 0.015 to $0.19 \mathrm{~g} / \mathrm{cm}^{3}$. The physical reason of this phenomenon is connected with formation of many particle clusters and requires further consideration. From Fig. 4 it can be easily seen that other predictions of PPT in hydrogen or hydrogenhelium mixtures $[2,30]$ with low mass concentration of helium are located in the beginning of the region where DPIMC simulation fails to converge. The sharp rise of electrical conductivity of hydrogen-helium mixture along the quasi-isentrope with the initial state $T=77.4 \mathrm{~K}$ and $P=8.1 \cdot 10^{-} 3 \mathrm{GPa}$ is also observed experimentally in the range of densities $0.5-0.83 \mathrm{~g} / \mathrm{cm}^{3}$ [35], see line with crosses in Fig. 4. Unfortunately the DPIMC in its current formulation can not give reliable information about the location and properties of PPT both in hydrogen and in hydrogen-helium plasmas. We plan to investigate these problems in our future work.

Acknowledgements This work is done under financial support of RF President Grant No. MK-1769.2003.08, the RAS program No. 17 "Parallel calculations and multiprocessor computational systems", the grant for talented young researchers of the Science support foundation and the Deutsche Forshungsgemeinschaft under grant BO 1366-2. The research was also partly sponsored by CRDF Awards and the Ministry of Education of Russian Federation.

\section{References}

[1] G. Chabrier, D. Saumon, W.B. Hubbard, J.I. Lunine, Astrophys. J. 391, 817-826 (1992).

[2] D. Saumon, G. Chabrier, H.M. Van Horn, Astrophys. J. Suppl. Ser. 99, 713-741 (1995).

[3] T.V. Gudkova, V.N. Zharkov, Planet. Space Sci. 47, 671-677 (2000).

[4] W.J. Nellis, Planet. Space Sci. 48, 671-677 (2000).

[5] D. Beule, W. Ebeling, A. Förster, H. Juranek, R. Redmer, G. Röpke, Journal de Physique IV 10, Pr5, 295-299 (2000).

[6] D. Saumon, G. Chabrier, Phys. Rev. A 44, 5122-5141 (1991).

[7] D. Saumon, G. Chabrier, Phys. Rev. A 46, 2084-2100 (1992).

[8] V.E. Fortov, V.Y. Ternovoi, M.V. Zhernokletov, M.A. Mochalov, A.L. Mikhailov, A.S. Filimonov, A.A. Pyalling, V.B. Mintsev, V.K. Gryaznov, I.L. Iosilevskii, JETP 97, 259-278 (2003).

[9] G.E. Norman, A.N. Starostin, Sov. Phys. High Temp. 6, 410 (1968).

[10] W. Ebeling, W.D. Kraeft, D. Kremp, Theory of Bound States and Ionization Equilibrium in Plasmas and Solids (Akademie-Verlag, Berlin, 1976).

[11] W.D. Kraeft, D. Kremp, W. Ebeling, G. Röpke, Quantum Statistics of Charged Particle Systems (Akademie, Berlin, 1986).

[12] V.M. Zamalin, G.E. Norman, V.S. Filinov, The Monte-Carlo Method in Statistical Thermodynamics (Nauka, Moscow, 1977).

[13] V.S. Filinov, M. Bonitz, V.E. Fortov, W. Ebeling, P. Levashov, M. Schlanges, Contrib. Plasma Physics 44, 400-406 (2004).

[14] V.S. Filinov, M. Bonitz, P.R. Levashov, V.E. Fortov, W.Ebeling, M. Schlanges, S.W. Koch, J. Phys. A.: Math. Gen. 36, 6069-6076 (2003).

[15] R.P. Feynman, Statistical Mechanics (Advanced Book Program, Reading, Massachusetts, 1972).

[16] G. Kalman, editor, Strongly Coupled Coulomb Systems (Pergamon, Oxford, 1988).

[17] W. Ebeling, H.J. Hoffmann, G. Kelbg, Contrib. Plasma Phys. 7, 233 (1967).

[18] A.V. Filinov, M. Bonitz, W. Ebeling, J. Phys. A: Math. Gen. 36, 5957-5962 (2003).

[19] V.S. Filinov, M. Bonitz, W. Ebeling, V.E. Fortov, Plasma Phys. Contr. Fusion 43, 743 (2001).

[20] V.S. Filinov, P.R. Levashov, V.E. Fortov, M. Bonitz, in: Progress in Nonequilibrium Green's Functions, edited by M. Bonitz, (World Scientific, Singapore, 2000), p. 513.

[21] V.S. Filinov, M. Bonitz, V.E. Fortov, JETP Letters 72, 245-248 (2000).

[22] V.S. Filinov, V.E. Fortov, M. Bonitz, D. Kremp, Phys. Lett. A 274, 228-235 (2000).

[23] V. Bezkrovniy, V.S. Filinov, D. Kremp, M. Bonitz, M. Schlanges, W.D. Kraeft, P.R. Levashov, V.E. Fortov, Phys. Rev. E 70, 057401 (2004).

[24] M.D. Knudson, D.L. Hanson, J.E.Bailey, C.A. Hall, J.R. Assay, Phys. Rev. Lett. 90, 035505 (2003).

[25] L.P. Da Silva, P. Celliers, et al., Phys. Rev. Lett. 78, 483-486 (1997).

[26] V.S. Filinov, V.E. Fortov, M. Bonitz, P.R. Levashov, JETP Letters 74, 384 (2001). 
[27] P.R. Levashov, V.S. Filinov, V.E. Fortov, M. Bonitz, in: Shock Compression of Condensed Matter — 2001, edited by M.D. Furnish, N.N. Thardhani, and Y.Horie (AIP, New York, 2002), p. 119.

[28] D. Saumon, and G. Chabrier, Phys. Rev. Lett. 62, 2397 (1989).

[29] X. Yan, S. Tsai, S. Ichimaru, Phys. Rev. A 43, 3057 (1991).

[30] M. Schlanges, M. Bonitz, A. Tschttschjan, Contrib. Plasma Phys. textbf35, 109-125 (1995).

[31] V.Y. Ternovoi, A.S. Filimonov, V.E. Fortov, S.V. Kvitov, D.N. Nikolaev, A.A. Pyalling, Physica B 265, 6-11 (1999).

[32] G.A. Thomas, T.-M. Rice, J.C. Hensel, Phys. Rev. Lett. 33, 219-222 (1974).

[33] S.T. Weir, A.C. Mitchell, W.J. Nellis, Phys. Rev. Lett. 76, 1860-1863 (1996).

[34] V.Y. Ternovoi, A.S. Filimonov, A.A. Pyalling, V.B.Mintsev, V.E. Fortov, in: Shock Compression of Condensed Matter — 2001, edited by M.D. Furnish, N.N. Thardhani, and Y.Horie (AIP, New York, 2002), p. 107.

[35] V.Y. Ternovoi, S.V. Kvitov, A.A. Pyalling, A.S. Filimonov, V.E. Fortov, JETP Letters 79, 8-11 (2004).

[36] W. Ebeling, A. F orster, V.E. Fortov, V.K. Gryaznov, A.Ya. Polishchuk, Thermophysical Properties of Hot Dense Plasmas (B.G. Teubner Verlagsgesellschaft, Stuttgart, Leipzig, 1991). 